\title{
Current Trends in Osteoporosis Diagnostics: An Opportunity for Quantitative Ultrasound and Biomarkers
}

\author{
Marnie M Saunders ${ }^{* 1}$, Sharon L Truesdell ${ }^{1}$, Estee L George ${ }^{1}$, James H Holda ${ }^{2}$ and Christopher Van \\ Vranken ${ }^{1}$ \\ ${ }^{1}$ Department of Biomedical Engineering, The University of Akron, USA \\ ${ }^{2}$ Department of Biology, The University of Akron, USA
}

*Corresponding author: Marnie M Saunders, Department of Biomedical Engineering, The University of Akron, Akron, OH 443250302, USA

\section{ARTICLE INFO}

Received: 幽 November 02, 2019

Published: 幽 November 19, 2019

Citation: Marnie M Saunders, Sharon L Truesdell, Estee L George, James H Holda, Christopher Van Vranken. Current Trends in Osteoporosis Diagnostics: An Opportunity for Quantitative Ultrasound and Biomarkers. Biomed J Sci \& Tech Res 23(1)-2019. BJSTR. MS.ID.003838.

Keywords: Osteoporosis; Diagnostics; Ultrasonics; Microfluidics; Point-of-Care

\begin{abstract}
Osteoporosis, characterized by diminished bone strength and increased susceptibility to fragility fracture, affects hundreds of millions throughout the world. Given the aging population, this number is only expected to grow. Currently dual x-ray absorptiometry (DXA) is the best tool for diagnosing osteoporosis; however, $75 \%$ of the affected global population remains undiagnosed and untreated. DXA scans give bone mineral density values that are indicative of bone strength. However, other risk factors must also be considered to accurately assess fracture probability. The World Health Organization released the fracture risk assessment algorithm (FRAX®) to accompany DXA scans and predict 10-year fracture risk, but the online tool better predicts those who will not suffer fragility fractures. Quantitative ultrasound can be used to assess bone microarchitecture and other properties leading to a better understanding of bone strength. Microfluidics can be used to measure bone turnover markers from small sample volumes. When coupled with point-of-care systems, these technologies can reach those in need. This review discusses these tools in advancing osteoporosis diagnostics and its subsequent treatment.
\end{abstract}

\begin{abstract}
Abbreviations: DXA: Dual X-ray Absorptiometry; FRAX: Fracture Risk Assessment Algorithm; BMD: Bone Mineral Density; PBM: Peak Bone Mass; BMC: Bone Mineral Content; BA: Bone Area; WHO: World Health Organization; BTM: Bone Turnover Marker; POC: Point-of-Care; ISCD: International Society for Clinical Densitometry; TBS: Trabecular Bone Score; VFA: Vertebral Fracture Assessment; QUS: Quantitative Ultrasound; SOS: Speed of Sound; BUA: Broadband Ultrasound Attenuation; PINP: Procollagen Type 1 N-Terminal Propeptide; CTX: C-terminal telopeptide, Collagen Type I; OC: Osteocalcin; NTX: N-Terminal telopeptide, Collagen Type I; Abs: Antibodies; Ags: Antigens; ELISA: Enzyme-Linked Immunosorbent Assay; PE: Pulse-Echo; DI: Density Index; FS: Fragility Score; EIS: Electrochemical Impedance Spectroscopy; LFA: Lateral Flow Assay
\end{abstract}

\section{Introduction}

Osteoporosis is a pathological decrease in bone mineral density (BMD) that increases risk of fragility fracture [1]. Osteoporosis affects 200 million people across the globe [2] and is underdiagnosed and undertreated [3]. The onset of osteoporosis is influenced by lifestyle choices, such as diet, exercise and tobacco use
[4-6], as well as additional risk factors, such as age and gender [1,6]. Postmenopausal women are particularly susceptible to the disorder and are generally advised to undergo routine examinations for bone health assessment. Given that emphasis is placed on diagnosing high-risk women, men are often overlooked [7]. The onset of 
osteoporosis may be predicted by peak bone mass (PBM), which is attained in the third decade of life $[8,9]$. Pediatricians suggest PBM accumulation, which is affected by several factors during growth, may be a powerful defense against development of osteoporosis later in life [10]. While preventative measures help reduce the risk of osteoporosis and associated fracture, prevention is not always an option. As such, diagnosis and treatment are needed to reduce morbidity and mortality and to increase quality of life.

Osteoporosis reduces bone mass, while bone cells work simultaneously to maintain a normal ratio of mineralized to nonmineralized matrix [1]. BMD is decreased when bone deviates from homeostasis and the rate of bone resorption exceeds the rate of bone formation. Osteoporosis is classified as either primary or secondary, where the former is senile or idiopathic [11] and the latter is due to drug intake, unloading or another disorder [12]. Bone mass is a significant determinant of fracture occurrence; thus, as BMD decreases, the risk of fragility fracture increases [11]. Osteoporosis often goes undetected until the onset of a fracture, and even then, diagnosis and treatment are not guaranteed $[13,14]$. One study involving over 2,000 women aged 50 years and older demonstrated that fragility fractures occurred in $81 \%$ of those who suffered a fracture. Moreover, the study reported that $79 \%$ of those individuals did not receive appropriate follow-up care or treatment for osteoporosis six to eight months following fracture [15]. The current gold standard for diagnosis of osteoporosis is by means of dual-energy X-ray absorptiometry (DXA) scanning [16].

The scan applies two distinct X-ray beams at the femoral head or lumbar region of the spine to obtain values for bone mineral content (BMC) and bone area (BA). From here, areal BMD can be calculated [17]. DXA requires specialized facilities, expensive laboratory equipment and highly trained medical personnel to operate and to calibrate the scanner $[18,19]$. Presently no technology can achieve the same clinical standard of osteoporotic diagnosis as DXA. However, it is estimated that $75 \%$ of the affected global population remains untreated [3]. As such, there is a significant need to develop more cost-effective and portable alternatives to DXA. The purpose of this review is to assess current bone health diagnostics and existing trends in the field. We discuss the globally accepted standards for measuring bone health-DXA and FRAX ${ }^{\circledR}$-as established by the World Health Organization (WHO). Additionally, we explore recent advancements in diagnosis of osteoporosis by means of ultrasound, microfluidic technologies, bone turnover markers (BTMs), and point-of-care (POC) systems. We conclude that, with further technological and research advancements, the future of bone health diagnostics will be based on quantitative microfluidics for the analysis of BTMs and quantitative ultrasound for the assessment of bone fragility.

\section{Current Bone Health Diagnostics}

\section{Dual-Energy X-Ray Absorptiometry}

Dual-Energy X-Ray Absorptiometry is the international standard diagnostic tool used to determine BMD [16]. As the name suggests, DXA scans use two X-ray beams of distinct energies: one to detect bone and the other to detect soft tissue. The scan measures BMC in grams and BA in square $\mathrm{cm}$; these measurements are usually taken at the femoral head or the lumbar region of the spine. Areal BMD-determined by dividing BMC by BA-gives a patient's T- and Z-score. The T- and Z-scores are interpreted by calculating standard deviations from the mean BMDs of young healthy adults of the same sex and healthy adults of the same age, respectively [17]. A T-score of - 1 or greater indicates normal BMD, a score between -1 and -2.5 indicates osteopenia, and a score of less than -2.5 indicates osteoporosis [1]. Osteoporosis is considered severe when risk of fragility fracture is also present [20]. When the scanners are maintained appropriately and operated by highly trained personnel, quality DXA reports can be generated. However, numerous studies have revealed that calibration, operation and maintenance of the equipment as well as interpretation of scans are highly variable [18,21].

In response the International Society for Clinical Densitometry (ISCD) released a set of guidelines titled "Best Practices for DualEnergy X-ray Absorptiometry Measurement and Reporting." The guidelines pertain to scan acquisition and analysis, as well as interpretation and reporting. The ISCD made numerous recommendations, including:

1) Spine phantom BMD measurements should be taken once a week to maintain a tolerance of $\pm 1.5 \%$,

2) At least one DXA technologist and one interpreter should have valid certifications in bone densitometry,

3) Each technologist should have completed an in vivo precision assessment,

4) The facility least significant change should be determined [19].

DXA scans require technologists, clinicians, interpreters and supervisors and results can vary greatly if these personnel are not proficient in recognizing confounding artifacts and other sources of error $[18,19]$. Use of DXA has certain disadvantages. Due to the size and complexity of the equipment, specialized facilities are required to house the scanners. Moreover, skilled medical personnel are necessary to operate and calibrate the device and interpret the results $[18,19]$. And, due to high demand, patients may have to wait long periods of time before scanners become available [22]. In summary, the resources required for operation of DXA scanners and their lack of portability render them inaccessible to much of the world.

\section{Trabecular Bone Score and Vertebral Fracture Assessment}

Several technologies exist that can be used alongside DXA to better identify persons at risk of fracture. Central DXA scans, more specifically those of the lumbar spine, can be further evaluated to determine a trabecular bone score (TBS). This value is computer software generated and provides an evaluation of overall bone microarchitecture. The TBS software estimates a 3D structure using 
the pixel differences from the 2D projection images. For postmenopausal women, a TBS above 1.350 indicates healthy microarchitecture, while a TBS below 1.200 indicates degraded microarchitecture. A TBS between these two values indicates microarchitecture that is partially degraded. No ranges for men have yet been established [23]. Several studies have demonstrated the utility of TBS in identifying patients at risk of vertebral fracture [23-25].

Another technology that utilizes central DXA is the vertebral fracture assessment (VFA). This lateral scan of the spine can be taken at the same time as DXA to examine the vertebrae for presence of fracture. The ability to run VFA in parallel reduces cost and radiation exposure; however, like DXA and traditional spinal radiographs, result interpretation relies on the expertise of the personnel performing the analysis [26,27]. In addition, VFA is performed with less sensitivity when paired with older densitometer models [26]. Both TBS and VFA have demonstrated utility in identifying persons at risk of vertebral fracture when used alongside DXA; however, neither solves the issues of availability or complexity of DXA scanners.

\section{Fracture Risk Assessment Algorithm and Garvan Fracture Risk Calculator}

Studies show that a large portion of individuals who suffer fragility fractures have BMD T-scores above the osteoporotic range and sometimes even in the healthy range [1]. This demonstrates that a low BMD is not the only fracture risk factor. As fragility fractures are the primary cause of morbidity and mortality among osteoporotic patients, it is imperative to be able to identify additional patient risk factors. It is for this reason the WHO developed a fracture risk assessment algorithm (FRAX ${ }^{\circledR}$ ) that incorporates femoral neck DXA BMD measurements and determines the 10-year probability of a patient 40 years or older suffering a fracture [28]. FRAX ${ }^{\circledR}$, available online, is nation-specific, as demographics alter fracture risk [29]. DXA measurements may be entered into the assessment tool, though they are not necessary for the calculation. To determine individual fracture risk FRAX ${ }^{\circledR}$ uses ten patient-specific factors, including age, sex, weight, height, previous fracture occurrence, tobacco use and femoral neck BMD (as determined by DXA) [29,30].

Studies demonstrate that $\mathrm{FRAX}^{\circledR}$ results in high specificity but low sensitivity in the diagnosis of osteoporosis [22,31]. In other words, the assessment tool adequately identifies patients without the disease but fails to identify a portion of patients with the disease. Therefore FRAX ${ }^{\circledR}$ is best used in conjunction with DXA to identify patients at risk. While this tool helps persons not at risk to avoid unnecessary and expensive tests, it may also provide a false sense of security to those in danger of suffering fragility fractures. Additionally, the tool may help lower screening costs, but it does not adequately identify those who require diagnosis and treatment. A similar tool is the Fracture Risk Calculator developed by Garvan Institute. The online tool incorporates four clinical risk factors and BMD for patients 50 years or older. These factors include age, sex, number of falls in last 12 months and fractures since the age of 50 [32]. While FRAX ${ }^{\circledR}$ was developed from data from 20 population-based cohorts throughout the world, the Garvan calculator was developed from the Dubbo Osteoporosis Epidemiology Study [32-34].

One New Zealand study evaluated both the FRAX ${ }^{\circledR}$ and Garvan calculators by conducting a 5 -year randomized, placebo-controlled study including over 1,400 postmenopausal women. BMD of the femoral neck was determined by DXA for each participant and they were asked to report fractures at 6-month intervals. The study determined that neither calculator had better predictive capabilities than models that incorporate only age and BMD. In addition, the discriminate ability of either tool was only moderate. The FRAX ${ }^{\circledR}$ calculator with BMD underestimated osteoporotic and hip fractures. When used without BMD, the tool underestimated osteoporotic fractures but overestimated hip fractures. The Garvan tool was well suited for osteoporotic fractures but overestimated hip fractures [32]. The inaccuracies of these tools put physicians and patients at risk of incorrect treatment.

\section{Ultrasound}

Quantitative ultrasound (QUS) methods are becoming a focus in osteoporosis management [35]. Ultrasound technology employs sound waves for noninvasive and non-ionizing diagnostics. These systems include piezoelectric transducers, called probes, which are usually applied to the skin, and generate sound waves and collect information from echoes. Relative to DXA, QUS systems are low-cost and more easily translated into portable machinery. QUS can assess bone microarchitecture and material properties, unlike DXA. Two QUS technologies are commonly used to assess bone: axial transmission and transverse transmission [35]. During axial transmission, both transducer types (transmitting and receiving) are placed on the same side of a long bone (e.g. radius [36] or tibia [37]), and ultrasound velocity is measured as it propagates across a known distance [35]. During transverse transmission, transducers are placed on either side of the bone of interest (e.g. calcaneus [38] or phalanges [39]) and measures the signal that transmits through the bone.

This technique can be used to determine speed of sound (SOS) or Broadband Ultrasonic Attenuation (BUA). Healthy bone is known to have greater velocity and higher attenuation. QUS is commonly restricted to the peripheral skeleton due to limited tissue interference; the calcaneus is often used [35]. A large prospective study including 15,000 women and men found that information gathered at the calcaneus from ultrasound can predict hip and total fracture risk [40]. In vitro studies have demonstrated a strong correlation exists between calcaneal BMD and SOS as well as between calcaneal BMD and BUA $[35,41]$. These findings demonstrate the high utility of QUS in bone health assessment. Several key disadvantages exist among current ultrasound techniques that must be overcome before they can surpass DXA 
technology in assessing bone health. There is significant variation across ultrasound technologies in how they are designed and operated. As such, two devices that make the same measurements may give different results. Additionally, many ultrasound systems have unconstrained transducers and non-automated protocols; measurement is largely operator dependent.

This prevents results from being reproducible, as shifted probe placement could result in a different measurement. Further, ultrasound results are largely affected by factors such as movement, temperature and soft tissue. Lastly there is no protocol established by the WHO to reduce variability among devices [35]. Ultrasound technology offers several advantages over DXA It is more cost effective, relatively portable and easier to operate and maintain $[35,42]$. As such, ultrasound technology may be incorporated into POC devices for osteoporosis detection. Presently no ultrasound technology meets DXA diagnostic standards in terms of sensitivity and specificity. However, studies suggest that many patients who suffer fragility fractures have T-scores that are above the osteoporotic range [1]. This indicates that while BMD is a major determinant of fracture risk, it is not the only determinant causing DXA to underrepresent those who require treatment. Thus, given its ability to estimate bone strength by assessment of bone microarchitecture, ultrasound technology may offer a key advantage over DXA.

\section{Bone Turnover Markers}

Bone turnover markers (BTMs) may be key to the success of POC bone health diagnostics. There are two categories of BTMs: bone formation markers and bone resorption markers. Bone formation markers are found in serum and plasma, and bone resorption markers are found in serum and urine [43]. While there are numerous BTMs in each category, only a few have been established as accurate correlators to BMD. The accepted bone formation and bone resorption markers are serum PINP (s-PINP) and serum CTX (s-CTX), respectively [44]. Bone formation marker, PINP, is the N-terminal propeptide of type I collagen. It is derived from the post-translational protease cleavage of procollagen molecules [43]. PINP, a monomer or trimer in serum, may be targeted using the automated Roche Elecsys or IDS-iSYS assays. The Roche Elecsys captures both the monomeric and trimeric forms while the IDSiSYS captures only the trimeric form [45]. These precise assays meet clinical standards because they produce low variability and remain stable at room temperature [44].

Osteocalcin (OC) is a late marker of osteoblast activity. It has been used in research; however, it is unstable, has a short halflife and is also influenced by circadian rhythm [43]. Despite its limitations in research, a negative correlation has been established between non carboxylated OC and hip fractures in postmenopausal women [46]. Bone resorption marker, s-CTX, is the C-terminal telopeptide of type I collagen. It is produced by the cleavage of type
I collagen by the cathepsin K enzyme. CTX exists in two isomerized forms, $\alpha$ and $\beta$. Following further isomerization, they are referred to as isomerized forms D and L, respectively [43]. While s-CTX has been established as a suitable marker for measuring bone resorption, the compound is known to be strongly susceptible to circadian variation [47]. It has been detected at maximum levels in the serum during mid-morning (05:00) and at minimum levels in the serum during mid-afternoon $(14: 00)[48,49]$. It is also greatly affected by food intake [43]. For these reasons, experts recommend samples be collected early in the morning after an evening of fasting and measurements be repeated with little environmental variation [50]. Bone resorption marker, urinary NTX (u-NTX), has also demonstrated great promise for use in clinical diagnostics. The marker is measured in urine samples which eliminates the need for medical personnel and laboratory instruments, increases its potential for use in a POC device and reduces patient discomfort and risk of infection. Further, NTX is less susceptible to circadian variations than CTX [43]. The major downside to the use of this BTM is that urine collection over several hours is necessary for testing [51]. BMD measurement using BTMs offers several key advantages. DXA and other imaging modalities give static measurements that cannot reveal a change in bone turnover rate. And a substantial amount of bone must be lost before those who interpret DXA results will categorize patients as high-risk and in need of treatment. On the other hand, BTM quantification requires a small sample volume and can indicate rate of change of bone turnover.

\section{Antibodies}

Antibodies (Abs) are key components in the capture and detection of BTMs. Abs are immunoglobulin proteins produced by the immune system in response to pathogens referred to as antigens (Ags). They are both highly specific and bind with high affinity. Specificity is a key characteristic of Abs which makes them ideal in the capture of BTMs. CTX from a human can be injected into a different species, such as a goat or rabbit. The protein will be recognized as a foreign $\mathrm{Ag}$, and an immune response will occur resulting in the production of anti-CTX ( $\alpha$-CTX) Abs. These same Abs (i.e. primary Abs) can then be injected into a different species to develop Abs against those Abs (e.g. anti $\alpha$-CTX Abs). These are referred to as secondary Abs. These secondary Abs can then have labels attached to them, such as gold particles or enzymes, making them readily detectible.

Both types of Abs may be utilized in the capture of peptides, proteins or hormones and for the development of appropriate clinical assays. Aptamers are single-stranded nucleic acids that carry the potential to replace $\mathrm{Ab}$ in the capture and measurement of BTMs [52,53]. Aptamers demonstrate high specificity and affinity (i.e. tight binding) like their Ab counterparts; however, they may be synthesized in the laboratory removing the need for animals [53]. In addition, aptamers have demonstrated greater stability than $\mathrm{Ab}$, as they are less susceptible to denaturing or losing their structure 
[54,55]. One group recently developed a novel DNA aptamer beacon to quantitate CTX and was able to bind the 26-amino acid CTX peptide [56]. The development of more BTM-specific aptamer sequences can offer greater stability of POC diagnostics for the assessment of bone health.

\section{Enzyme-Linked Immunosorbent Assays}

Enzyme-linked immunosorbent assays (ELISAs) are widely used laboratory tests that utilize primary and secondary Abs in the capture and quantitation of BTMs. For simplification, BTMs will be referred to as Ags for the remainder of this section. ELISAs, commonly performed in 96-well plates, use detection enzymes or other labels for Ag quantification. There are several types of ELISAs: direct, indirect and sandwich. The sandwich technique is able to detect very low concentrations of $\mathrm{Ag}$ and thus applies to detection of BTMs. In a sandwich ELISA, the bottom of each well is coated with capture Abs specific to the Ag of interest. The sample is added, and time is allowed for Ab-Ag binding. Next a detection $\mathrm{Ab}$ also specific to the Ag is added. This Ab may be unlabeled, or enzyme linked. If the detection $\mathrm{Ab}$ is unlabeled, an enzyme-linked secondary $\mathrm{Ab}$ is added. Lastly, substrate is added and cleaved by the enzyme which results in a color signal.

To determine Ag concentration, a standard curve is run concurrently with the sample. Microplate readers are used to measure color intensity after reactions have occurred. ELISAs require small sample volumes for quantification of Ag present. This makes them highly useful in clinical settings for the detection of BTMs in serum. However, there are several key aspects of ELISAs that make them unsuitable for POC application. ELISAs require trained personnel in a clinical setting to perform the assays properly and microplate readers to measure the results. The enzyme/substrate reaction is rapid, and results must be read quickly. Further, the tests require hours to perform, and occasionally an overnight coating process. ELISAs are not able to be translated into POC devices for bone health diagnostics; however, concepts from the sandwich ELISA are used in the lateral flow assay discussed below.

\section{Future of Bone Health Diagnostics}

Current standards for bone health diagnostics include DXA which is costly and unavailable to most of the world, as well as FRAX $^{\circledR}$ which is most suitable for identifying persons not at risk for fracture than those at risk. POC technologies assess bone microarchitecture through QUS and bone turnover rate through quantitation of BTMs in microfluidics. QUS assessment of bone microarchitecture estimates bone strength better than DXA. Microfluidics, small-scale tools that can measure BTMs in small sample volumes, are used to indicate bone remodeling rates, while DXA gives a static measurement. Abs have been used to capture BTMs. As science works to move away from the use of animals due to ethics and cost, aptamers offer a promising alternative to
Abs. In order to close the osteoporosis care gap, the field should incorporate these advancements in technology.

\section{Point-of-Care}

The two most promising technologies for bone health point-ofcare (POC) diagnostics are QUS and microfluidics. QUS, while most commonly used to measure the peripheral skeleton when central fractures are most debilitating has been shown to accurately assess bone properties that may be correlated to fracture risk [57-59]. Microfluidic and similar BTM-detection technologies have been developed to rapidly quantify BTMs at lower costs and without the need for large laboratory equipment [60-62].

\section{POC Ultrasound}

Several QUS technologies have been developed and tested for use in osteoporosis diagnosis. Pulse-echo (PE) ultrasound has demonstrated great promise in helping to identify persons at risk of fragility fractures. PE ultrasound is used in the determination of density index (DI) [63], which is highly correlated with the BMD of the femoral neck [64]. PE focuses on the thickness of the cortical bone as it is known to diminish in osteoporotic bone $[65,66]$. This thickness is used in combination with the age, height and weight of the patient to calculate a DI [64]. While this measurement cannot perfectly identify patients at risk of osteoporosis (roughly $30 \%$ of patients require verification with other diagnostics), it represents a significant improvement over calcaneal ultrasound and peripheral DXA. Calcaneal QUS and peripheral DXA result in 56\% and 39-56\% accuracy, respectively [63]. PE ultrasound does not yet measure up to central DXA; however, its portability may lead to its eventual use in osteoporosis POC devices.

Another use of ultrasound technology in bone health assessment is the development of a parameter called the fragility score (FS). A recent study compared this parameter to spinal DXA to determine how well it could identify patients at risk of fragility fractures. The ultrasound scan was performed on the abdomen and the resulting images of the lumbar vertebrae were used to calculate the FS. The images were processed using a novel algorithm that assigns a FS to a patient using spectral and statistical analyses. According to the study, the score demonstrated sensitivity of $76 \%$ and specificity of $68 \%$ which is slightly better than the BMD as determined by DXA [42] (Table 1). Other ultrasound measurements for bone health include apparent integrated backscatter [67], broadband ultrasound backscatter [57], frequency dependent backscatter coefficient [68,69], integrated reflection coefficient [64], mean of backscatter difference spectrum [58], osteoporosis score [59] and slope of backscatter difference spectrum [58]. No ultrasound technology has yet to parallel DXA's ability to accurately diagnose osteoporosis; its potential for identifying patients at risk of fragility fracture and translation into POC systems makes its continual advancements vital (Table 1 ). 
Table 1: Ultrasound technologies for bone assessment.

\begin{tabular}{|c|c|c|c|c|}
\hline Ultrasound Technology & Measurement & Validation & Result & Ref \\
\hline $\begin{array}{l}\text { Apparent Integrated } \\
\text { Backscatter (AIB) }\end{array}$ & Femoral head ex vivo & micro -CT & $\begin{array}{l}\text { Correlation of } \mathrm{r}=0.69 \text { between AIB and trabecular } \\
{ }^{\mathrm{a}} \mathrm{BV} / \mathrm{TV} \text { with compensation for sample-probe } \\
\text { distance and cortical thickness }\end{array}$ & [67] \\
\hline $\begin{array}{l}\text { Broadband Ultrasound } \\
\text { Backscatter (BUB) }\end{array}$ & $\begin{array}{l}\text { Distal femur and proximal } \\
\text { tibia ex vivo }\end{array}$ & Bone mechanical properties & $\begin{array}{c}\text { Significant correlation of backscatter parameters } \\
\text { with ultimate strength of trabecular bone and bone } \\
\text { volume fraction }(r=0.90)\end{array}$ & [57] \\
\hline Fragility Score (FS) & $\begin{array}{l}\text { Abdominal scan of lumbar } \\
\text { vertebrae in vivo }\end{array}$ & Spinal DXA & $\begin{array}{l}\text { F.S. performed slightly better than DXA at identifying } \\
\text { persons at risk of fragility fracture } \\
\text { Sensitivity/Specificity: } \\
\text { Ultrasound: } 76 / 68 \% \\
\text { DXA: } 73 \% / 66 \%\end{array}$ & [42] \\
\hline $\begin{array}{l}\text { Frequency Dependent } \\
\text { Backscatter Coefficient } \\
\text { (BSC) }\end{array}$ & $\begin{array}{l}\text { Bovine proximal femur } \\
\text { trabecular bone samples } \\
\text { ex vivo }\end{array}$ & $\begin{array}{l}\text { Apparent bone density } \\
\text { using a balance and a mass } \\
\text { cylinder }\end{array}$ & $\begin{array}{l}\text { Significant correlation between apparent bone } \\
\text { density and backscatter coefficient } \\
(0.5 \mathrm{MHz}: \mathrm{r}=0.751,1.0 \mathrm{MHz}: \mathrm{r}=0.808)\end{array}$ & [69] \\
\hline $\begin{array}{l}\text { Integrated Reflection } \\
\text { Coefficient (IRC) }\end{array}$ & $\begin{array}{l}\text { Proximal femur, } \\
\text { Proximal radius, } \\
\text { Proximal and distal tibia } \\
\text { in vivo }\end{array}$ & Axial DXA & $\begin{array}{l}\text { Cortical thickness at distal \& proximal tibia with } \\
\text { patient age and weight gave significant BMD } \\
\text { Sensitivity: } 86 \% \text {, Specificity: } 100 \%\end{array}$ & [64] \\
\hline $\begin{array}{l}\text { Mean of Backscatter } \\
\text { Difference Spectrum } \\
\text { (MBD) }\end{array}$ & $\begin{array}{l}\text { Cube-shaped specimens } \\
\text { of human cancellous bone } \\
\text { ex vivo }\end{array}$ & $\begin{array}{l}\text { Density measurements } \\
\text { with digital calipers and } \\
\text { electronic scale }\end{array}$ & $\begin{array}{l}\text { Moderate to good correlations between backscatter } \\
\text { difference spectrum frequency averaged mean and } \\
\text { cancellous density }(\mathrm{r}=0.70-0.95)\end{array}$ & [58] \\
\hline Osteoporosis Score (OS) & $\begin{array}{l}\text { Abdominal echographic } \\
\text { scanning of lumbar spine } \\
\text { in vivo }\end{array}$ & Spinal DXA & $\begin{array}{l}\text { OS diagnosis is } 91.1 \% \text { accurate compared to DXA. } \\
\text { Significant correlations between OS-estimated BMD } \\
\text { and DXA BMD } \\
\text { (R up to } 0.73 \text { and RMS error of } 6.3 \%-9.3 \%)\end{array}$ & [59] \\
\hline $\begin{array}{l}\text { Slope of Backscatter } \\
\text { Difference Spectrum } \\
\text { (SBD) }\end{array}$ & $\begin{array}{l}\text { Cube-shaped specimens } \\
\text { of human cancellous bone } \\
\text { ex vivo }\end{array}$ & $\begin{array}{l}\text { Density measurements } \\
\text { with digital calipers and } \\
\text { electronic scale }\end{array}$ & $\begin{array}{l}\text { Weak to moderate correlations between backscatter } \\
\text { difference spectrum frequency slope and cancellous } \\
\text { density }(r=0.21-0.80)\end{array}$ & [58] \\
\hline
\end{tabular}

Note: ${ }^{\mathrm{B}} \mathrm{BV} / \mathrm{TV}$ : Bone volume fraction.

\section{Microfluidic and BTM-Detection Technologies}

Microfluidic technology is used to develop POC devices for the diagnosis of osteoporosis. Microfluidic technologies are highly specific and sensitive, cost effective and easily made portable; they also require small sample volumes. Further the systems can operate without the need for trained medical personnel. Multiplex microfluidics have the ability to detect multiple analytes in a single system. They are a focus in bone health diagnostics as research has found that more than one protein is needed to gain a full understanding of physiologic conditions [43]. Yun et al. [60] developed a label-free electronic biosensor that measures BTMs using electrochemical impedance spectroscopy (EIS). The system can be used for multiplexing without the need for secondary labels. According to the researchers, based on the methodology, a wide array of proteins can be measured by simply incorporating different Abs into the system. They began with the detection of CTX by applying a self-assembled monolayer of dithiodipropionic acid to a gold electrode which served as the working electrode in the system.

Biotinylated Abs specific to CTX were immobilized on the surface using streptavidin as it has a high binding affinity for biotin. EIS responses proved to increase with Ag concentration. The researchers achieved a detection limit of $30 \mathrm{ng} / \mathrm{mL}$ and a dynamic range of $3 \mu \mathrm{g} / \mathrm{mL}$ [60] (Table 2). More recently Khashayar et al. [61] published proof-of-concept of a multiplex microfluidic platform for the detection of BTMs. The group used electrochemical sensing to determine BTM concentrations from a serum sample. Gold electrodes were fabricated in a microfluidic chamber and manipulated to immobilize Abs specific to CTX and OC. The system enables capture of the two BTMs and measures oxidative peaks that can then be converted to concentrations. This method measures current response which has been shown to correlate linearly with the logarithmic concentration of the BTM. The group achieved limits of detection of $1.39 \mathrm{pg} / \mathrm{mL}$ and $1.94 \mathrm{ng} / \mathrm{mL}$ for CTX and OC, respectively [61] (Table 2).

Based on technology of the sandwich ELISA, Lee et al. [62] developed a lateral flow immunoassay (LFA) for the detection of s-CTX and u-NTX. BTMs were captured using Abs without the use of electronic biosensors. LFA strips were fabricated by combining a treated sample pad, a conjugate pad with dried and labeled Abs, a nitrocellulose analytical membrane, a wicking pad to collect 
excess fluids, and a backing material for the entire strip (Figure 1). Anti-CTX and anti-NTX Abs were immobilized on the analytical membrane and secondary Abs for each were immobilized further down the strip (i.e. test and control line). Sample containing Ag was applied to the sample pad which then wicked to the conjugate pad. This remobilized Abs specific to the Ag of interest that were conjugated to colloidal gold and had been previously dried onto the conjugate membrane. All contents then wicked to the analytical membrane. The present Ag was sandwiched between the Abs from the conjugate pad and those present on the analytical membrane.

The gold-Ab conjugate bound to Ag present in the sample produced a color signal at the test and control lines that could be correlated to $\mathrm{Ag}$ concentration. The researchers used a digital camera and ImageJ software to quantify the results. The findings were compared to commercial NTX and CTX ELISAs and demonstrated a correlation of 0.913 and 0.872 , respectively [62] (Table 2) (Figure 1). Microfluidic technologies offer great potential for the development of POC devices for bone health assessment. These devices are more cost effective than DXA and allow for the detection of multiple BTMs within small sample volumes. The scale, cost and simplicity of these technologies means their commercialization could reach far more osteoporotic patients at risk of fragility fracture than any other bone diagnostic tool available. To our knowledge, none of these devices have been commercialized but published findings demonstrate the field is moving in the right direction.

Table 2: Microfluidic technologies for quantifying bone turnover markers.

\begin{tabular}{|c|c|c|c|c|}
\hline Microfluidic Technology & BTM(s) & Validation & Results & Ref \\
\hline $\begin{array}{l}\text { Label-Free Electronic Biosensor using } \\
\text { Electrochemical Impedance Spectroscopy }\end{array}$ & СTX & $\begin{array}{l}\mathrm{Ag} / \mathrm{Agcl} \text { reference } \\
\text { electrode }\end{array}$ & $\begin{array}{l}\text { Limit of Detection: } 50 \mathrm{ng} / \mathrm{mL} \\
\text { Dynamic Range: up to } 3 \mu \mathrm{g} / \mathrm{mL}\end{array}$ & [60] \\
\hline $\begin{array}{l}\text { Multiplex Microfluidic Platform with } \\
\text { Electrochemical Sensing }\end{array}$ & $\begin{array}{c}\text { s-CTX; } \\
\text { Osteocalcin }\end{array}$ & ${ }^{a}$ ECLIA & $\begin{array}{c}\text { Limits of Detection: s-CTX: } 1.39 \mathrm{pg} / \mathrm{mL} \\
\text { Osteocalcin: } 1.94 \mathrm{ng} / \mathrm{mL} \\
\text { Dynamic Range: s-CTX: } 25-1008 \mathrm{pg} / \mathrm{ML} \\
\text { Osteocalcin: } 9-42 \mathrm{ng} / \mathrm{ML}\end{array}$ & [61] \\
\hline Lateral Flow Immunoassay & s-CTX; u-NTX & ELISA & $\begin{array}{l}\text { Correlation of LFA to ELISA: s-CTX: } 0.872 \\
\text { u-NTX: } 0.913 \\
\text { Precision of LFA to ELISA: s-CTX: } 0.995 \\
\text { u-NTX: } 0.974\end{array}$ & [62] \\
\hline
\end{tabular}

Note: aECLIA: Electrochemiluminescence immunoassay.

Fluid Flow $\rightarrow$

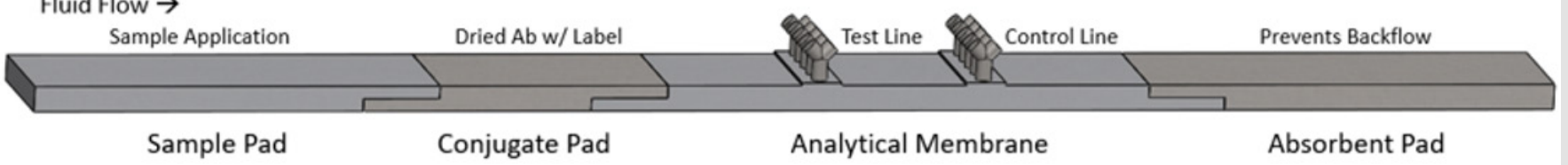

Figure 1: Basic lateral flow (LFA) assembly. The LFA assembly consists of a treated sample pad, conjugate pad, analytical nitrocellulose membrane and an absorbent pad. The materials overlap to allow fluid to wick through the device. Proteins specific to the target analyte are present on the conjugate pad and at the test and control lines.

\section{Discussion}

Osteoporosis characterized by low BMD and increased risk of fragility facture affects hundreds of millions of individuals worldwide most of whom have not been diagnosed. The WHO established DXA and nation specific FRAX ${ }^{\circledR}$ as standards for osteoporosis diagnosis and fracture risk assessment. These tools, while the best available are insufficient. DXA scans are expensive and subject to variation and they require operation by skilled technicians. Additionally, the technology is not suitable for translation into POC systems. FRAX ${ }^{\circledR}$, while more attainable is best suited for identifying persons not at risk of fracture. Ultrasound and microfluidic technologies can determine bone properties and remodeling rates, respectively. However, automated ultrasound systems and assessment protocols must be developed to overcome variability in measurements. Microfluidic systems must be adapted to be multiplex and give results that can be interpreted easily and cheaply. While there is currently no cure for osteoporosis there are several measures that can be taken to delay its progression and reduce risk of fracture.

Parents of adolescents who are aware of osteoporosis within their genealogy can help their children take known preventative measures to enhance PBM accumulation. For example, children should consume diets high in calcium, receive adequate doses of vitamin D [4] and take part in physical activities that include impact- and muscle-loading [70]. Furthermore, parents can warn children that tobacco use $[6,71,72]$ and inadequate nutrition [73] can prove damaging to the skeleton in the long term. Similar preventative measures should be taken by adults who have surpassed the age at which bone mass peaks. Adults should also ensure adequate calcium intake and vitamin D exposure $[74,75]$ and avoid tobacco 
use $[4,71]$. Osteoporotic patients may be treated with bisphosphonates to inhibit bone resorption and maintain bone mass [1]. Other treatments exist and may be more appropriate for certain patients; however, osteoporosis is most commonly treated with bisphosphonates [75]. Preventative measures and therapy are critical for those at risk of osteoporotic fracture; however, diagnosis remains a global challenge on which research efforts should be focused.

\section{Conclusion}

Osteoporosis debilitates hundreds of millions of people across the globe. There should be an emphasis during childhood education on lifestyle choices that can prevent onset of the disease. In addition, diagnosis and treatment need to be improved. For example, standardized protocols and automation are needed to enhance QUS. A better understanding needs to be developed for the interpretation of BTM measurements. To that end, a microfluidic POC device capable of quantifying BTMs and novel QUS technology that can accurately establish bone properties carry great promise.

\section{Acknowledgement}

M Crow was monetarily compensated for contributions to this manuscript. This work was supported by the National Science Foundation [EBMS 1700299]. This work is posthumously dedicated to Dr James Holda (1954-2019).

\section{Conflict of Interest}

Marnie Saunders, Sharon Truesdell, Estee George, James Holda and Christopher Van Vranken declare that they have no conflict of interest.

\section{References}

1. Kanis JA (1994) Organization, World Health. Assessment of fracture risk and its application to screening for postmenopausal osteoporosis: report of a WHO study group [meeting held in Rome from 22 to 25 June 1992]. Osteoporos Int 4(6): 368-381.

2. Reginster JY, Burlet N (2006) Osteoporosis: a still increasing prevalence. Bone 38(2Suppl 1): S4-9.

3. Nguyen TV, Center JR, Eisman JA (2004) Osteoporosis: underrated, underdiagnosed and undertreated. Med J Aust 180(S5): S18-22.

4. Kitchin B, Morgan SL (2007) Not just calcium and vitamin D: other nutritional considerations in osteoporosis. Curr Rheumatol Rep 9(1): 85-92.

5. Krall EA, Dawson Hughes B (1993) Heritable and life-style determinants of bone mineral density. JBMR 8(1): 1-9.

6. Nguyen T, Kelly P, Sambrook P, Gilbert C, Pocock N, et al. (1994) Lifestyle factors and bone density in the elderly: implications for osteoporosis prevention. JBMR 9(9): 1339-1346.

7. Papaioannou A, Kennedy CC, Ioannidis G, Gao Y, Sawka A, et al. (2008) The osteoporosis care gap in men with fragility fractures: The Canadian Multicentre Osteoporosis Study. Osteoporos Int 19(4): 581-587.

8. Lu J, Shin Y, Yen MS, Sun SS (2016) Peak bone mass and patterns of change in total bone mineral density and bone mineral contents from childhood into young adulthood. J Clin Densitom 19(2): 180-191.

9. Recker RR, Davies KM, Hinders SM, Heaney RP, Stegman MR, et al. (1992) Bone gain in young adult women. JAMA 268(17): 2403-2408.
10. Gordon CM, Zemel BS, Wren TA, Leonard MB, Bachrach LK, et al. (2017) The determinants of peak bone mass. J Pediatr 180: 261-269.

11. Cooper C (1999) Epidemiology of osteoporosis. Osteoporos Int 9(8): S2S8.

12. Miazgowski T, Kleerekoper M, Felsenberg D, Štěpán JJ, Szulc P (2012) Secondary osteoporosis: endocrine and metabolic causes of bone mass deterioration. J Osteoporos 2012: 907214.

13. Leslie W, Giangregorio L, Yogendran M, Azimaee M, Morin S, et al. (2012) A population-based analysis of the post-fracture care gap 1996-2008: the situation is not improving. Osteoporos Int 23(5): 1623-1629.

14. Flais J, Coiffier G, Le Noach J, Albert J, Faccin M, et al. (2017) Low prevalence of osteoporosis treatment in patients with recurrent major osteoporotic fracture. Arch Osteoporos 12(1): 24.

15. Bessette L, Ste Marie LG, Jean S, Davison K, Beaulieu M, et al. (2008) The care gap in diagnosis and treatment of women with a fragility fracture. Osteoporos Int 19(1): 79-86.

16. Roux C, Briot K (2017) Current role for bone absorptiometry. Joint Bone Spine 84(1): 35-37.

17. Berger A (2002) Bone mineral density scans. BMJ 325(7362): 484.

18. Morgan SL, Prater GL (2017) Quality in dual-energy X-ray absorptiometry scans. Bone 104: 13-28.

19. Lewiecki EM, Binkley N, Morgan SL, Shuhart CR, Camargos BM, et al. (2016) Best practices for dual-energy X-ray absorptiometry measurement and reporting: International Society for Clinical Densitometry Guidance. J Clin Densitom 19(2): 127-140.

20. Kanis J, Glüer CC (2000) An update on the diagnosis and assessment of osteoporosis with densitometry. Osteoporos Int 11(3): 192-202.

21. Lewiecki EM, Lane NE (2008) Common mistakes in the clinical use of bone mineral density testing. Nat Rev Rheumatol 4(12): 667-674.

22. Cherian KE, Kapoor N, Shetty S, Naik D, Thomas N, et al. (2018) Evaluation of different screening tools for predicting femoral neck osteoporosis in rural South Indian postmenopausal women. J Clin Densitom 21(1): 119124.

23. Silva BC, Leslie WD, Resch H, Lamy O, Lesnyak O, et al. (2014) Trabecular bone score: a noninvasive analytical method based upon the DXA image. JBMR 29(3): 518-530.

24. Briot K, Paternotte S, Kolta S, Eastell R, Reid DM, et al. (2013) Added value of trabecular bone score to bone mineral density for prediction of osteoporotic fractures in postmenopausal women: the OPUS study. Bone 57(1): 232-236.

25. Iki M, Fujita Y, Tamaki J, Kouda K, Yura A, et al. (2015) Trabecular bone score may improve FRAX® prediction accuracy for major osteoporotic fractures in elderly Japanese men: the Fujiwara-kyo osteoporosis risk in men (FORMEN) cohort study. Osteoporos Int 26(6): 1841-1848.

26. Zeytinoglu M, Jain RK, Vokes TJ (2017) Vertebral fracture assessment: Enhancing the diagnosis, prevention, and treatment of osteoporosis. Bone 104: 54-65.

27. Jager P, Jonkman S, Koolhaas W, Stiekema A, Wolffenbuttel B, et al. (2011) Combined vertebral fracture assessment and bone mineral density measurement: a new standard in the diagnosis of osteoporosis in academic populations. Osteoporos Int 22(4): 1059-1068.

28. Kanis JA, Oden A, Johansson H, Borgström F, Ström O, et al. (2009) FRAX and its applications to clinical practice. Bone 44(5): 734-743.

29. Kanis J, Mc Closkey E, Johansson H, Oden A, Ström O, et al. (2010) Development and use of FRAX in osteoporosis. Osteoporos Int 21(Suppl 2): S407-413.

30. Kanis JA, Borgstrom F, De Laet C, Johansson H, Johnell O, et al. (2005) Assessment of fracture risk. Osteoporos Int 16(6): 581-589. 
31. Cummins NM, Poku E, Towler M, O’Driscoll OM, Ralston S (2011) Clinical risk factors for osteoporosis in Ireland and the UK: a comparison of FRAX and QFractureScores. Calcif Tissue Int 89(2): 172-177.

32. Bolland MJ, Siu AT, Mason BH, Horne AM, Ames RW, et al. (2011) Evaluation of the FRAX and Garvan fracture risk calculators in older women. JBMR 26(2): 420-427.

33. Kanis J, Odén A, Johnell O, Johansson H, De Laet C, et al. (2007) The use of clinical risk factors enhances the performance of BMD in the prediction of hip and osteoporotic fractures in men and women. Osteoporos Int 18(8): 1033-1046.

34. Nguyen ND, Frost SA, Center J, Eisman JA, Nguyen TV (2008) Development of prognostic nomograms for individualizing 5-year and 10-year fracture risks. Osteoporos Int 19(10): 1431-1444

35. Laugier P (2004) An overview of bone sonometry. International Congress Series 1274: 23-32.

36. Hans D, Srivastav S, Singal C, Barkmann R, Njeh C, et al. (1999) Does combining the results from multiple bone sites measured by a new quantitative ultrasound device improve discrimination of hip fracture? JBMR 14(4): 644-651.

37. Foldes A, Rimon A, Keinan D, Popovtzer M (1995) Quantitative ultrasound of the tibia: a novel approach for assessment of bone status. Bone 17(4): 363-367.

38. Langton C, Palmer S, Porter R (1984) The measurement of broadband ultrasonic attenuation in cancellous bone. Eng Med 13(2): 89-91.

39. Duboeuf F, Hans D, Schott AM, Giraud S, Delmas PD, et al. (1996) Ultrasound velocity measured at the proximal phalanges: precision and age-related changes in normal females. Revue du rhumatisme (English Edi.) 63(6): 427-434.

40. Khaw KT, Reeve J, Luben R, Bingham S, Welch A, et al. (2004) Prediction of total and hip fracture risk in men and women by quantitative ultrasound of the calcaneus: EPIC-Norfolk prospective population study. Lancet 363(9404): 197-202.

41. Tsuda Futami E, Hans D, Njeh C, Fuerst T, Fan B, et al. (1999) An evaluation of a new gel-coupled ultrasound device for the quantitative assessment of bone. BJR 72(859): 691-700.

42. Pisani P, Greco A, Conversano F, Renna MD, Casciaro E, et al. (2017) A quantitative ultrasound approach to estimate bone fragility: A first comparison with dual X-ray absorptiometry. Measurement 101: 243 249.

43. Shetty S, Kapoor N, Bondu JD, Thomas N, Paul TV (2016) Bone turnover markers: emerging tool in the management of osteoporosis. Indian Endocrinol Met 20(6): 846-852.

44. Vasikaran S, Eastell R, Bruyère O, Foldes A, Garnero P, et al. (2011) Markers of bone turnover for the prediction of fracture risk and monitoring of osteoporosis treatment: a need for international reference standards. Osteoporos Int 22(2): 391-420.

45. Brandt J, Krogh TN, Jensen CH, Frederiksen JK, Teisner B (1999) Thermal instability of the trimeric structure of the N-terminal propeptide of human procollagen type I in relation to assay technology. Clin Chem 45(1): 47-53

46. Iftikhar A, Asim T, Islam AU, Ahmed ST (2018) Association of serum P1NP (N-terminal propeptide of Type1 collagen) and bone mineral density (BMD) in osteoporosis. Pakistan Armed Forces Medical Journal 68(2): 357-362.

47. Chubb SP (2012) Measurement of C-terminal telopeptide of type I collagen (CTX) in serum. Clin Biochem 45(12): 928-935.

48. Hannon R, Eastell R (2000) Preanalytical variability of biochemical markers of bone turnover. Osteoporos Int 11(Suppl 6): S30-S44.

49. Qvist P, Christgau S, Pedersen B, Schlemmer A, Christiansen C (2002) Circadian variation in the serum concentration of $\mathrm{C}$-terminal telopeptide of type I collagen (serum CTx): effects of gender, age, menopausal status, posture, daylight, serum cortisol, and fasting. Bone 31(1): 57-61.
50. Clowes J, Hannon R, Yap T, Hoyle N, Blumsohn A, et al. (2002) Effect of feeding on bone turnover markers and its impact on biological variability of measurements. Bone 30(6): 886-890.

51. Garnero P, Sornay Rendu E, Claustrat B, Delmas PD (2000) Biochemical markers of bone turnover, endogenous hormones and the risk of fractures in postmenopausal women: the OFELY study. JBMR 15(8): 1526-1536.

52. Ellington AD, Szostak JW (1990) In vitro selection of RNA molecules that bind specific ligands. Nature 346(6287): 818-812.

53. Kinghorn AB, Fraser LA, Lang S, Shiu SCC, Tanner JA (2017) Aptamer Bioinformatics. Int J Molec Sci 18(12): pii E2516.

54. Sharma TK, Bruno JG, Dhiman A (2017) ABCs of DNA aptamer and related assay development. Biotech Adv 35(2): 275-301.

55. Catanante G, Mishra RK, Hayat A, Marty JL (2016) Sensitive analytical performance of folding based biosensor using methylene blue tagged aptamers. Talanta 153: 138-144.

56. Bruno JG, Carrillo MP, Phillips T, Hanson D, Bohmann JA (2011) DNA aptamer beacon assay for C-telopeptide and handheld fluorometer to monitor bone resorption. J Fluoresc 21(5): 2021-2033.

57. Karjalainen JP, Töyräs J, Riekkinen O, Hakulinen M, Jurvelin JS (2009) Ultrasound backscatter imaging provides frequency-dependent information on structure, composition and mechanical properties of human trabecular bone. Ultrasound Med Biol 35(8): 1376-1384.

58. Hoffmeister BK, Wilson AR, Gilbert MJ, Sellers ME (2012) A backscatter difference technique for ultrasonic bone assessment. J Acoust Soc Amer 132(6): 4069-4076.

59. Conversano F, Franchini R, Greco A, Soloperto G, Chiriacò F, et al. (2015) A novel ultrasound methodology for estimating spine mineral density. Ultrasound Med Biol 41(1): 281-300.

60. Yun YH, Bhattacharya A, Watts NB, Schulz MJ (2009) A label-free electronic biosensor for detection of bone turnover markers. Sens $9(10)$ : 7957-7969.

61. Khashayar P, Amoabediny G, Larijani B, Hosseini M, Verplancke R, et al. (2017) A multiplexed microfluidic platform for bone marker measurement: A proof-of-concept. Micromachines 8(5): 133.

62. Lee KM, Lee MH, Chung CY, Seong WK, Lee SD, et al. (2012) Measurement of urinary $\mathrm{N}$-telopeptides and serum C-telopeptides from type I collagen using a lateral flow-based immunoassay. Sens 13(1): 165-174

63. Karjalainen J, Riekkinen 0, Töyräs J, Jurvelin J, Kröger H (2016) New method for point-of-care osteoporosis screening and diagnostics. Osteoporos Int 27(3): 971-977.

64. Karjalainen J, Riekkinen O, Töyräs J, Hakulinen M, Kröger H, et al. (2012) Multi-site bone ultrasound measurements in elderly women with and without previous hip fractures. Osteoporos Int 23(4): 1287-1295.

65. Wear KA (2013) Autocorrelation and cepstral methods for measurement of tibial cortical thickness. IEEE Transactions on Ultrasonics, Ferroelectrics, and Frequency Control 50(6): 655-660.

66. Karjalainen J, Riekkinen O, Toyras J, Kroger H, Jurvelin J (2008) Ultrasonic assessment of cortical bone thickness in vitro and in vivo. IEEE Transactions on Ultrasonics, Ferroelectrics, and Frequency Control 55(10): 2291-2297.

67. De Marco T, Peccarisi M, Conversano F, Greco A, Chiozzi S, et al. (2016) A new approach for measuring the trabecular bone density through the echosound backscattering: An ex vivo validation on human femoral heads. Measurement 87: 51-61.

68. Wear KA, Nagaraja S, Dreher ML, Gibson SL (2012) Relationships of quantitative ultrasound parameters with cancellous bone microstructure in human calcaneus in vitro. J Acoust Soc Amer 131(2): 1605-1612.

69. Il Lee K, Joo Choi M (2012) Frequency-dependent attenuation and backscatter coefficients in bovine trabecular bone from 0.2 to $1.2 \mathrm{MHz}$. J Acoust Soc Amer 131(1): EL67-EL73. 
70. Gunter KB, Almstedt HC, Janz KF (2012) Physical activity in childhood may be the key to optimizing lifespan skeletal health. Exer Sport Sci Rev 40(1): 13-21.

71. Law M, Hackshaw A (1997) A meta-analysis of cigarette smoking, bone mineral density and risk of hip fracture: recognition of a major effect BMJ 315(7112): 841-846.

72. Ward KD, Klesges RC (2001) A meta-analysis of the effects of cigarette smoking on bone mineral density. Calcif Tissue Int 68(5): 259-270.

ISSN: 2574-1241

DOI: 10.26717/BJSTR.2019.23.003838

Marnie M Saunders. Biomed J Sci \& Tech Res

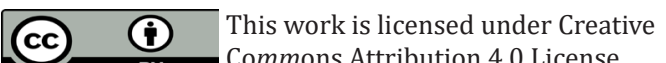

Submission Link: https://biomedres.us/submit-manuscript.php
73. Bachrach LK, Guido D, Katzman D, Litt IF, Marcus R (1990) Decreased bone density in adolescent girls with anorexia nervosa. Pediatr 86(3): 440-447.

74. Tang BM, Eslick GD, Nowson C, Smith C, Bensoussan A (2007) Use of calcium or calcium in combination with vitamin $\mathrm{D}$ supplementation to prevent fractures and bone loss in people aged 50 years and older: a meta-analysis. Lancet 370(9588): 657-666.

75. Chan CK, Mason A, Cooper C, Dennison E (2016) Novel advances in the treatment of osteoporosis. BMB 119(1): 129-142.

$\begin{array}{ll}\text { BIOMEDICAL } & \text { Assets of Publishing with us } \\ \text { RESEARCHES } & \text { - Global archiving of articles } \\ & \text { - Immediate, unrestricted online access } \\ \end{array}$

